\title{
Assistência de enfermagem ao paciente com lesão renal aguda: relato de experiência
}

\author{
Nursing care to patients with acute kidney injury: experience report \\ Cuidados de enfermería a pacientes con lesión renal aguda: informe de experiencia
}

Recebido: 19/08/2021 | Revisado: 24/08/2021 | Aceito: 26/08/2021 | Publicado: 29/08/2021

\author{
Keyla Bispo Silva \\ ORICID: https://orcid.org/0000-0003-3308-3124 \\ Faculdade Irecê, Brasil \\ E-mail: bispokeyla6@gmail.com \\ Fernanda Abade Lemos \\ ORICID: https://orcid.org/0000-0001-9062-1270 \\ Faculdade Irecê, Brasil \\ E-mail: fernandalemos222@outlook.com \\ Uanderson Gomes dos Santos \\ ORICID: https://orcid.org/0000-0002-5641-8030 \\ Faculdade Irecê, Brasil \\ E-mail: uandergomesz@outlook.com \\ Jomara de Souza Dourado \\ ORICID: https://orcid.org/0000-0002-2681-3099 \\ Faculdade Irecê, Brasil \\ E-mail: jomara.dourado@hotmail.com
}

\begin{abstract}
Resumo
Objetivo geral: Identificar como deve ocorrer a assistência de enfermagem frente ao paciente com LRA no ambiente intra-hospitalar. Metodologia: Trata-se de um estudo, descritivo/bibliográfico/qualitativo, do tipo relato de experiência realizada no ano de 2019, no estágio supervisionado II, realizado em uma Unidade Hospitalar de pequeno porte. Resultados e Discussões: A comparação da experiência vivenciada, pôde evidenciar que grande parte dos enfermeiros desconhece ou não prioriza o processo de enfermagem, o que contribui para deteriorização da função renal e consequentemente piora do prognóstico do paciente. Considerações finais: Destaca-se a importância da realização de educação permanente dos profissionais de enfermagem, o qual, auxiliará os mesmos a manusearem o processo de enfermagem, garantindo cuidado integral aos pacientes, contribuindo para diminuição da mortalidade pela LRA.
\end{abstract}

Palavras-chave: Lesão renal aguda; Enfermagem; Assistência de enfermagem.

\begin{abstract}
General objective: To identify how nursing care should be provided to patients with AKI in the intra-hospital environment. Methodology: This is a descriptive/bibliographical/qualitative study, of the experience report type carried out in 2019, in the supervised internship II, performed in a small hospital unit. Results and Discussions: The comparison of the lived experience could show that most nurses do not know or do not prioritize the nursing process, which contributes to the deterioration of renal function and consequently worsens the patient's prognosis. Final considerations: The importance of continuing education of nursing professionals is highlighted, which will help them to handle the nursing process, ensuring integral care to patients, contributing to the reduction of mortality by AKI.
\end{abstract}

Key words: Acute kidney injury; Nursing; Nursing care.

\section{Resumen}

Objetivo general: Identificar cómo debe producirse la asistencia enfermera frente al paciente con ERS en el ambiente intrahospitalario. Metodología: Se trata de un estudio, descriptivo/bibliográfico/cualitativo, del tipo relato de experiencia realizado en el año 2019, en el estadio supervisado II, realizado en una Unidad Hospitalaria de pequeño porte. Resultados y discusiones: La comparación de la experiencia vivida pudo demostrar que la mayoría de las enfermeras no conocen o no priorizan el proceso de enfermería, lo que contribuye al deterioro de la función renal y, en consecuencia, empeora el pronóstico del paciente. Consideraciones finales: Se destaca la importancia de la formación permanente de los profesionales de enfermería, que les ayudará a manejar el proceso enfermero, garantizando la atención integral a los pacientes, contribuyendo a la reducción de la mortalidad por IRA.

Palabras clave: Lesión renal aguda; Enfermagem; Asistencia de enfermería. 


\section{Introdução}

A lesão renal aguda (LRA) é considerada como uma patologia que apresenta uma gama de sintomas clínicos, caracterizada por declínio abrupto da função renal, o qual pela diminuição da taxa de filtração glomerular provoca acúmulo de metabólicos, levando a alteração no equilíbrio hidroeletrolítico e acidobásico no organismo. No entanto, apesar de ser considerada como uma das patologias mais frequentes no ambiente intra-hospitalar, a LRA possui elevada chance de reversibilidade, se diagnosticada e tratada precocemente (Levi, Souza, Magalhães, Carvalho, Cunha, Dantas, Cruz, Guimarães \& Crus, 2013).

Segundo Melo, Pereira, Saldanha, Alves e Sousa (2015) a prevalência de LRA, aumentou na última década em países desenvolvidos. Esses autores afirmam ainda, que o aumento dessa prevalência decorre do envelhecimento populacional, o qual atrelado a indivíduos com múltiplas comorbidades, leva ao uso de múltiplas drogas e consequentemente o declínio da função renal. Vale ressaltar que, não só os medicamentos trazem malefícios à função renal, diversas comorbidades, como o diabetes e hipertensão arterial sistêmica, possui grande potencial de ocasionar deteriorização renal, sendo as principais causas da insuficiência renal crônica (IRC).

A LRA possui elevada letalidade, sendo responsável pela morte de aproximadamente 2 milhões de pessoas por ano (Li, Burdmann \& Mehta, 2013). Embora nas últimas décadas o avanço tecnológico e científico tenha contribuído para criação de formas mais precoces e fidedignas de diagnóstico e tratamento, a taxa de mortalidade por LRA permanece relativamente constante, girando em torno de 50\%, o que demonstra que além do avanço cientifico, deve-se investir ainda mais na assistência realizada por meio da mão de obra humana (Peres, Junior, Schafer, Silva, Gaspar, Scarpari, Alves, Neto \& Oliveira, 2013).

Assim, objetivando a diminuição da mortalidade, bem como das complicações ocasionadas pela LRA no ambiente hospitalar é que a enfermagem como integrante da equipe multidisciplinar, deve participar ativamente do plano de cuidados oferecidos ao paciente diagnosticado com LRA. Os mesmos declaram ainda, que as ações devem estar pautadas no conhecimento técnico e científico, e que a assistência de enfermagem deve ser realizada de modo ágil e preciso, a fim de contribuir para melhor prognóstico ao paciente (Silva, Pires, Pereira, Andrade, Leite, Góes, oliveira, Gonzaga, Guidi \& Pompeu, 2017).

Corroborando com esses autores, Martins e Silva (2016) relatam que embasados nos conhecimentos científicos, bem como nos sinais, sintomas e complicações advindas da LRA, o enfermeiro deve estabelecer um plano de cuidados utilizando para isso ferramentas essenciais do seu processo de trabalho. Essas ferramentas incluem a Sistematização da assistência de enfermagem (SAE) e a classificação de intervenções de enfermagem (NIC).

Dessa forma, ao se ater desses métodos, o enfermeiro após a realização do processo de anamnese e exame físico, terá dados que poderão classificar se o paciente está incluso em algum grupo de risco para o desenvolvimento da patologia, e caso já esteja acometido pela LRA, esse profissional poderá indicar os diagnósticos de enfermagem relacionados a essa patologia, e a partir do planejamento das ações, poderá executar as ações de forma qualificada, contribuindo para prevenir as complicações da LRA e ainda impedir a progressão da doença (Cerqueira, Tavares \& Machado, 2014).

Assim, diante da elevada taxa de mortalidade e, buscando-se registrar informações que possam contribuir para guiar o enfermeiro em sua prática, é que se elencou o seguinte objetivo: identificar como deve ocorrer a assistência de enfermagem frente ao paciente com LRA no ambiente intra-hospitalar, comparando as ações registradas na literatura com a experiência vivenciada em uma unidade hospitalar de pequeno porte.

Trata-se ainda de uma pesquisa de cunho qualitativo, do tipo relato de experiência, presenciado no estágio supervisionado II, realizado em uma Unidade Hospitalar de pequeno porte, localizada em um município do Estado da Bahia. Além do registro da experiência vivenciada, utilizou-se para fundamentar essa pesquisa, informações contidas em artigos e dissertações, publicados entre o período de 2013 a 2021, encontrados nos bancos de dados digitais. 


\section{Metodologia}

O presente estudo tem como foco a utilização de algumas ferramentas para promover o delineamento da pesquisa. Nesse sentido, adotou-se como estratégia metodológica a característica descritiva, a qual é definida por Gil (2010) como o método que possibilita a descrição das individualidades de determinada população ou fenômeno. Trata-se ainda de um de uma pesquisa de cunho qualitativo do tipo relato de experiência, onde segundo o Instituto Ciências da Vida (2016) é definido como o tipo de pesquisa que permite a descrição do autor, sobre uma situação vivenciada, sendo ela exitosa ou não, e que possibilite a discussão e a troca de ideias no meio científico.

Os fatos mencionados nesse estudo foram presenciados no terceiro trimestre do ano de 2019, durante 35 dias do estágio supervisionado II, ciclo I, realizado em uma Unidade Hospitalar de pequeno porte, localizada em um município do Estado da Bahia. Dessa forma, ao vivenciar em prática curricular a assistência de enfermagem ofertada a 05 pacientes diagnosticados com Lesão Renal Aguda (LRA), é que se elencou como objetivo descrever as práticas vivenciadas, fazendo uma correlação com o disposto na literatura.

Fora utilizado o método de revisão bibliográfica, cujo objetivo se deu em observar e contextualizar os assuntos elencados nos artigos relacionados à assistência de enfermagem frente a pacientes com LRA. Para esta finalidade foram selecionadas informações registradas em bancos de dados como: scielo, bireme, lilacs e dissertações e protocolos de unidades hospitalares.

Como critérios de inclusão foram utilizados artigos que tratavam sobre a LRA e que abordavam o papel do enfermeiro no diagnóstico, estratificação de risco e tratamento dessa patologia, bem como artigos publicados entre o período de 2013 a 2021, usando para tal os seguintes descritores: Insuficiência Renal Aguda, Lesão Renal Aguda, enfermagem, assistência de enfermagem, sendo selecionado após esse momento 23 artigos para leitura. Como critério de exclusão, foram deletados os artigos e materiais que possuíam idioma divergente do português e que não correlacionavam a LRA com a enfermagem.

A extração de dados foi baseada no método exploratório, a qual teve como finalidade identificar qual a relevância dos artigos, utilizando para análise dos dados, a leitura seletiva. Além desses, também foi instituído o método de leitura analítica, a qual teve por intento analisar e resumir as informações que se julgou necessário conter essa pesquisa. Todavia para compor essa pesquisa, foi utilizado ainda, o diário de bordo, o qual possibilitou explanar e descrever de forma clara e objetiva a experiência vivenciada em campo de estágio, permitindo correlacionar com exatidão os fatos vivenciados com as informações registradas na literatura.

\section{Resultados e Discussão}

Devido as diferentes classificações da LRA, definidas de acordo a patologia que precipitou o comprometimento da função renal, o paciente poderá apresentar sinais e sintomas inespecíficos, o que retarda e dificulta seu diagnóstico. Entretanto, apesar das diferentes manifestações clínicas de acordo a classificação da LRA, podendo ser pré-renal, intra-renal e pós-renal, o indivíduos podem apresentar sintomas específicos como febre, mialgia, redução do débito urinário em $50 \%$ dos casos, náuseas, anorexia, dor abdominal, alteração cognitiva, confusão mental, convulsão, coma, congestão pulmonar, edema agudo de pulmão, edema periférico, ascite e hiponatremia (Miarelli, Frezza \& Okino, 2017).

A LRA, é ainda responsável por ocasionar a diminuição da taxa de filtração glomerular, levando ao aumento da concentração de ureia e creatinina sérica ou ainda a presença de oligúria ou anúria. Por conseguinte, as repercussões do acúmulo de escórias nitrogenadas, pode ocasionar encefalopatia, neuropatia e pericardite, já o aumento do volume intravascular, devido à presença de oligúria ou anúria, pode ocasionar dispneia, edema pulmonar e distúrbios hidroeletrolíticos, sendo necessário em alguns casos a realização de diálise (Polini, Jahnke \& Rotta, 2020). 
Constituído como membro da equipe multidisciplinar, o papel do enfermeiro vai desde a participação para estabelecer o diagnóstico da LRA, bem como participante ativo das medidas terapêuticas para a patologia. Nesse sentido, sabendo-se que a detecção precoce da patologia, contribui para menores taxas de mortalidades e complicações, é que, em uso do processo de enfermagem, o enfermeiro deve através da anamnese identificar se o paciente possui patologias que representam um risco para o comprometimento da função renal, sendo elas comorbidades como diabetes, hipertensão, insuficiência cardíaca, infarto agudo do miocárdio, diarreia, hemorragia, grandes queimaduras, sepse, uso de medicamentos nefrotóxicos, uso de contraste, e intoxicações (Benichel \& Meneguin, 2020).

Entretanto, apesar de Soares, Silqueira, Marques e Souza (2019) registrarem em sua pesquisa que o profissional de enfermagem, a partir do uso do processo de enfermagem é responsável junto com a equipe em detectar os fatores de risco, ou ainda os sintomas sugestivos de LRA, durante a experiência vivenciada na unidade hospitalar, foi evidenciado que o enfermeiro não participa do plano de cuidados que visa detectar precocemente a LRA pela estratificação de risco do paciente, ficando ao profissional médico, a atribuição de realizar a suspeição e diagnóstico da LRA, bem como de implementar medidas terapêuticas.

Conforme registrado na literatura, os enfermeiros que atuam na emergência, terapia intensiva ou no internamento hospitalar, não possuem conhecimento satisfatório para executar o processo de avaliação do paciente, param assim se realizar estratificação de risco, prevenção e diagnóstico da LRA. Assim, conforme registrado na literatura, e analisado na unidade hospitalar utilizada como campo para essa pesquisa, a falta de conhecimento dos enfermeiros sobre os sinais e sintomas da LRA é que impede a realização de uma assistência satisfatória, capaz de regredir a disfunção renal, o que culmina em pior prognóstico para o paciente (Grassi, Dell'Acqua, Jenses, Fontes \& Guimarães, 2017).

Além de contribuir no diagnóstico, os profissionais de enfermagem devem também estar inseridos no plano de cuidados terapêuticos do paciente com LRA, contribuindo para menor período de internação, bem como diminuição da mortalidade hospitalar por LRA. Nesse sentido, após o diagnóstico, prioriza-se como terapêutica, a manutenção e correção da depleção volêmica, sendo utilizada para essa ação o balanço hídrico, o qual possibilita a monitorização das perdas e ingestas e infusões de líquidos no paciente (Lopes, Schran, Oliveira, Oliveira \& Fernandes, 2018).

Entretanto, apesar de ser constituído como umas das ferramentas indispensáveis para guiar as ações ofertadas ao paciente com LRA, na unidade hospitalar em questão, os enfermeiros não realizavam essa monitorização, e em casos de extrema necessidade registrava-se apenas as perdas hídricas do paciente em uma folha indevidamente adaptada, o qual, não garantia a monitorização fidedigna do balanço hídrico, impossibilitando a manutenção volêmica adequada ao paciente.

A monitoração de parâmetros hemodinâmicos, bem como dos sinais vitais também são atribuições dos profissionais, devendo segundo Alves e Cavalcante (2017), ser realizadas e registradas diariamente de acordo a necessidade do paciente, conforme prescrição médica. Vale ressaltar que pacientes oligúricos, com presença de edema, é necessário que haja monitorização hemodinâmica invasiva ou não invasiva, garantindo que qualquer alteração cardíaca ou circulatória seja detectada para assim se evitar complicações.

Nessa continuidade, tendo em vista que a unidade hospitalar de pequeno porte não possuía equipamentos especializados para a realização de monitorização hemodinâmica invasiva, e tendo em vista que os pacientes diagnosticados com LRA durante a experiência não possuíam indicação para esse tipo de ação, a abordagem utilizada consistia na monitorização hemodinâmica não invasiva, sendo essa realizada e registrada de maneira fidedigna pelos profissionais.

Considerando-se que a sepse é considerada como uma das patologias que precipita e piora o prognóstico dos indivíduos já diagnosticados com LRA, uma das funções do enfermeiro no ambiente hospitalar, consiste na prevenção ou detecção precoce de focos de infecções, utilizando para isso a adoção de técnicas assépticas para realização de procedimentos 
invasivos, monitorar sinais sugestivos de infecção e ainda realizar higienização adequada das mãos, evitando infecções cruzadas (Santos \& Marinho, 2013).

Contudo, apesar da instituição prezar pela realização das medidas de prevenção de infecção hospitalar foi perceptível o descuido dos profissionais de enfermagem quando a realização de medidas assépticas para prevenção das infecções. Nesse sentido, foi notória a negligência da higienização correta das mãos, e além da não realização das etapas para essa ação, os profissionais em sua maioria não se atentam a realização dessa ação antes e após de realizar algum procedimento no paciente, o que aumenta substancialmente o risco de infecção cruzada, podendo ocasionar pior prognóstico aos pacientes com LRA.

Tendo em vista o aumento do catabolismo ocasionado pela LRA, o enfermeiro também possui atribuição de monitorar o estado nutricional do paciente, realizando para isso pesagem diária sempre que possível, para que qualquer alteração seja detectada e solucionada. Entretanto, apesar da literatura indicar o enfermeiro como participante ativo do estado nutricional do paciente, na unidade hospitalar em questão, essa monitorização é realizada pelo profissional nutricionista, ficando a cargo do enfermeiro observar se o paciente está aceitando a dieta ofertada, ou se o mesmo possui necessidade de utilizar dispositivo para alimentação enteral por meio de sonda nasogástrica ou nasoenteral, sendo atribuído ao médico a prescrição da instalação desses dispositivos (Cardoso, 2014).

Além dessas alterações, atenção especial deve ser direcionada aos aspectos neurológicos dos pacientes com LRA, sendo segundo Silva e Martins (2017) atribuição do enfermeiro a monitorização de sinais e sintomas sugestivos de comprometimento neurológico devido ao acúmulo de compostos nitrogenados como a ureia. Dessa forma, atenção deve ser dada a pacientes que apresentam alteração no nível de consciência, déficit cognitivo, associado á cefaleia, alterações visuais, tremores, crises epiléticas ou até mesmo coma profundo, sintomas esses associados à encefalopatia urêmica.

Todavia, durante a experiência, apesar de prescrito pelo médico a realização de neuro-check conforme a necessidade dos indivíduos com LRA, alguns profissionais de enfermagem relataram desconhecer essa ferramenta, portanto, não realizavam essa avaliação. Vale ressaltar ainda, que apesar de um dos pacientes diagnosticados com LRA apresentar sinais sugestivos de comprometimento neurológico, os profissionais não correlacionavam essas alterações a LRA, o que impossibilitou uma abordagem específica para reverter o comprometimento neurológico (Moreira, Matta, Melo, Kummer, Barbosa, Teixeira, Simões \& Silva, 2014).

Além dos sinais e sintomas específicos da LRA, o paciente pode apresentar complicações como: o comprometimento da pele, com aparecimento de lesões de pressão, devido à presença de edema; distúrbios eletrolíticos; edema agudo de pulmão e encefalopatia. Dessa forma, as ações de prevenção para essas complicações consistem em: mudança de decúbito de duas em duas horas; avaliação de exames laboratoriais, detectando alteração de eletrólitos, bem como de substâncias como creatinina e ureia; se atentar a necessidade de balanço hídrico negativo, a fim de evitar hipervolemia em pacientes oligúricos; e realizar neuro-check conforme prescrição médica (Gomes \& Papaléo, 2015).

Ressalta-se como estratégia de extrema relevância, a realização do balanço hídrico em pacientes com risco para desenvolver LRA durante o período de internação hospitalar. Esse por sua vez, além de ser um instrumento de fácil aplicação, permite monitorizar a função renal através do registro de ganhos e perdas hídricas, sendo utilizado para mensurar o volume de líquido administrado e o volume excretado pelo paciente, sendo um marcador sensível para detecção precoce de LRA (Dantas, Vieira, Oliveira, Araújo \& Maximiano, 2021).

Diante das diversas complicações a serem prevenidas, cabe ao profissional de enfermagem à realização de exame físico e neurológico minucioso, para além de possibilitar a detecção de alterações, possa auxiliar na construção de cuidados para o paciente com LRA. Vale ressaltar, que a literatura afirma ainda, que para garantir destreza dessas ações, o profissional de enfermagem deve se ater da ferramenta do processo de enfermagem, elaborando para cada paciente um plano de cuidados 
que garanta tanto a prevenção de complicações, como a realização de terapêutica eficaz (Melo, Barbosa, Fernandes, França, Araújo, Abreu, Marques, Silveira, Rocha \& Araújo, 2017).

Todavia, apesar do conhecimento de sua importância, os enfermeiros na unidade hospitalar utilizada para essa pesquisa não faziam uso correto dessa ferramenta, o que impedia a realização do diagnóstico de enfermagem, bem como o planejamento de ações e das intervenções utilizadas para cada paciente, contribuindo para pior prognóstico do mesmo.

Vale ressaltar, que além de não implementar o processo de enfermagem, o desconhecimento dos sinais e sintomas da LRA, contribui para não realização dos planos de cuidados específicos para esses pacientes, pois para elaboração das intervenções necessárias, além do conhecimento técnico, o enfermeiro deve possuir conhecimento cientifico, levantando assim os diagnósticos possíveis para essa patologia, bem como prevenindo as complicações através da detecção dos riscos a qual o paciente está exposto.

Assim, ao fazer uma relação do que é proposto como atribuição da enfermagem na literatura, com o que foi vivenciado em prática, possibilitou concluir que os profissionais de enfermagem, ainda encontram uma deficiência no que diz respeito à realização das ações que devem ser executadas por ele frente aos pacientes com LRA, o que deixa esses pacientes sem a assistência necessária, contribuindo direta e indiretamente para aumento do período de hospitalizações, maiores chances de complicações, progressão da disfunção renal e consequentemente o óbito do paciente.

\section{Considerações Finais}

Apesar da assistência de enfermagem ser considerada como prática que contribui para redução de complicações e óbitos ocasionados pela LRA, pôde-se evidenciar após a experiência vivenciada que, ainda há desconhecimento por parte dos profissionais de enfermagem, quanto as ações que devem ser realizadas para prevenir, identificar ou ainda efetivar uma terapêutica eficaz ao paciente acometido por LRA.

Dessa forma, tendo em vista a elevada incidência dessa patologia no ambiente hospitalar, e sabendo-se que a participação ativa da enfermagem possibilita melhores prognósticos ao paciente, propõe-se a execução de atividades de educação permanente dos profissionais de enfermagem, para que os mesmos tenham respaldo para realizar desde a identificação da LRA, até a prescrição dos cuidados de enfermagem, possibilitando assistência individualizada e continua ao paciente.

Ressalta-se ainda, que embora o processo de enfermagem seja constituído como ferramenta que auxilia a prática da equipe de enfermagem e que garante maior autonomia a esses profissionais, observa-se dificuldade e comodismo para execução fidedigna dessa ferramenta, o que culmina em resultados insatisfatórios na assistência. Portanto conforme proposto, o processo de educação permanente, além de transmitir informações clínicas sobre a LRA, auxiliará os enfermeiros a manusear de forma correta essa ferramenta, garantindo cuidado integral a cada paciente, contribuindo para diminuição da mortalidade por essa patologia.

Assim, tendo em vista os prejuízos ocasionados pela LRA, e sabendo-se que essa se enquadra como um problema de saúde pública, enfatiza-se a necessidade da execução de estudos posteriores, tendo como objetivo abordar com exatidão os riscos, sinais e sintomas iniciais dessa patologia, tendo como foco ainda, abordar como a assistência de enfermagem, pautada no processo de enfermagem, enquadra-se como instrumento de extrema relevância para cuidar desses pacientes, tendo como objetivo, diminuir direta e indiretamente o número de mortes ocasionadas por essa doença.

\section{Referências}

Alves, G. F., \& Cavalcante, A. A. (2017). Atuação de enfermagem em pacientes com insuficiência renal aguda (IRA). Anais da Mostra de Pesquisa em Ciência e Tecnologia. Ceará, ISSN: 2238-2208. 
Benichel, C. R., \& Meneguin, S. (2020). Fatores de risco para lesão renal aguda em pacientes clínicos intensivos. Acta Paulista enferm. Vol. 33, ISSN19820194 .

Cardoso, S. S. (2014). Avaliação nutricional de pacientes com lesão renal aguda de um hospital de ensino. Faculdade de medicina de São José do Rio Preto. http://bdtd.famerp.br/handle/tede/323.

Cerqueira, D. P., Tavares, J. R., \& Machado, R. C. (2014). Fatores preditivos da insuficiência renal e algoritmo de controle e tratamento. Revista Latino Americano enfermagem, mar.-abr. 2014; 22(2):211-7.

Dantas, L. A. L., Vieira, A. N., Oliveira, L. C., Araújo, M. E. S., \& Maximiano, L. C. S. (2021). Fatores de risco para Lesão Renal Aguda em Unidades de Terapia intensiva. Research, Society and Development, 10(6), e32210615700, 2021.

Gil, A. C. (2010). Como elaborar projetos de pesquisa. Editora atlas, (5 edição).

Gomes, E. T., \& Papaléo, M. M. M. (2015). Caracterização dos pacientes portadores de insuficiencia renal atendidos na emergência de um hospital geral. Revista de enfermagem da universidade estadual do Rio de Janeiro. Rio de Janeiro, 23(4), 2015.

Grassi, M. F., Dell'Acqua, M. C. Q., Jenses, R., Fontes, C. M. B., \& Guimarães, H. C. Q. C. P. (2017). Diagnósticos, resultados e intervenções de enfermagem em pacientes com lesão renal aguda. Acta Paul Enferm. São Paulo, 2017; 30(5):538-45.

Instituto Ciências da Vida. (2016). Instrutivo para elaboração de relato de experiência. Universidade Federal de Juiz de Fora. Minas Gerais.

Levi, T. M., Souza, S. P., Magalhães, J. G., Carvalho, M. S., Cunha, A. L. B., Dantas, J. G. A. O., Cruz, M. G., Guimarães, Y. L. M., \& Crus, C. M. S. (2013). Comparação dos critérios RIFLE, AKIN e KDIGO quanto á capacidade de predição de mortalidade em pacientes graves. Revista de Terapia Intensiva. Artigo original, 25(4):290-296

Li, P. K. T., Burdman, E. A., \& Mehta, R. L. (2013). Injúria Renal Aguda: uma alerta global. Comitê Gestor do dia Mundial do Rim. Scielo, ;35(1):1-5.

Lopes, D., Schran, L. S., Oliveira, J. L. C., Oliveira, R. B. S. R. O., \& Fernandes, L. M. (2018). Fatores de risco/causais para insuficiência renal aguda em adultos internados na terapia intensiva. Enfermagem Brasil 2018;17 (4): 336-46.

Martins, J., \& Silva, S. N. S. (2016). Assistência de enfermagem ao paciente com insuficiência renal aguda em unidade de terapia intensiva. Instituto Nacional de Ensino Superior e Pesquisa. Monografias do Curso de MBA CCHI, Jacareí.

Melo, E. M., Barbosa, A. S., Fernandes, L. N. A., França, N. S., Araújo, S. S., Abreu, M. A. F., Marques, A. M., Silveira, F. M. M., Rocha, M. P., \& Araújo, R. M. R. P. (2017). Fatores preditivos de lesão renal aguda em pacientes internados em unidade de terapia intensiva. Revis. Tenden. Da Enferm. Profis. 2017; 9 (1); 2083-2088.

Melo, W. F., Pereira, A. W. R., Alves, V. Q., Saldanha, H. G. A. C., \& Sousa, J. S. (2015). Assistência de enfermagem na urgência e emergência ao paciente vítima de insuficiência renal aguda: uma revisão bibliográfica. Revista Brasileira de educação e saúde. ISSN 2358-2391- Pombal-PB, Brasil, 5(2), 06-11.

Miarelli, C. P., Frezza, G., \& Okino, V. T. (2017). Lesão renal Aguda. Revista da Faculdade de Medicina de Ribeirão Preto.

Moreira, J. M., Matta, S. M., Melo e Kummer, A., Barbosa, I. G., Teixeira, A. L., \& Simões e Silva, A. C. (2014). Transtornos neuropsiquiátricos e doenças renais: uma atualização. Artigo de atualização, Scielo, 2014;36(3):396-400.

Peres, L. A. B., Junior, A. D. C., Schafer, A. J., Silva, A. L., Gaspar, A. D., Scarpari, D. F., Alves, J. B. F., Neto, R. G., \& Oliveira, T. F. T. (2013). Biomarcardores da Injúria renal aguda. Artigo de Revisão. Scielo, 2013;35(1):1-5.

Poloni, J. A. T., Jahnke, V. S., \& Rotta, L. N. (2020). Insuficiência Renal Aguda em pacientes com COVID-19. Revista Brasileira de Analises Clínicas. ISSN: 2448-3877.

Santos, E. S., \& Marinho, C. M. S. (2013). Principais causas de insuficiência renal aguda em unidades de terapia intensiva: intervenção de enfermagem. Revista de enfermagem, 3(9), março, 2013, 181-189.

Silva, A. A., Pires, F. L., Pereira, K. C., Andrade, L. C. V., Leite, L. M., Góes, M. A., Oliveira, V. F. S., Gonzaga, M. F. N., Guidi, L. R., \& Pompeu, L. C. (2017). O processo de enfermagem (PE)- Sistematização da assistência de enfermagem (SAE) no paciente com insuficiência renal. Revista Saúde em focoEdição $\mathrm{n}^{\circ} 9$.

Silva, G. G. O., Nunes, J. T., Barboza, I. R., Barros, T. R. C. C. R., Souza, A. M. L., Davim, R. M. B., \& Martino, M. M. F. (2017). Distúrbios renais em unidade de terapia intensiva. Revista de enfermagem UFPE. Recife; 11(11): 4463-4468, nov.2017.

Soares, B. M. R., Silqueira, L. B., Marques, T. P. D., \& Souza, A. V. (2019). Histórico de enfermagem: instrumento para identificação de fatores de risco e prevenção de Lesão Renal Aguda em Unidade de Terapia Intensiva. Revista Univale, ISSN: 2358-2200. 